\title{
François de la Noue (1531-1591) au service du libéralisme du XIXe siècle
}

WILLIAM H. HUSEMAN

Il ne semble guère nécessaire de présenter le maréchal François de La Noue aux lecteurs de cette revue. Si La Noue est demeuré un personnage moins célèbre que Coligny, Monluc, Condé, et d'Aubigné, l'on aurait tort de sous-estimer le rôle qu'a joué le "Bras de Fer" dans les guerres de religion et dans l'"institution" de la Réforme en France. ${ }^{1}$ La parution en 1967 d'une édition moderne de ses Discours politiques et militaires ${ }^{2}$ a contribué à l'accroissement de sa réputation en rendant accessible à la communauté des seizièmistes une oeuvre riche mais relativement peu connue. Depuis cette date, de nombreux chercheurs ont recouru à son témoignage à cause de la lucidité de ses analyses et de la qualité indéniable de sa prose. ${ }^{3}$ Le lecteur des Discours découvre avec plaisir non pas les réminiscences décousues d'un vieux guerrier hargneux mais un témoignage équitable qui impressionne par sa franchise et sa bonne foi, qualités rarissimes à l'époque des guerres de religion. L'on comprend facilement pourquoi Montaigne a pu s'émerveiller de "la constante bonté, douceur de meurs et facilité conscientieuse de monsieur de la Nouë, en une telle injustice de parts armees, vraie eschole de trahison, d'inhumanité et de brigandage, où tousjours il s'est nourry, grand homme de guerre et très-experimenté."4 Serait-il superflu de rappeler ici que la plupart des historiens se sont rangés du côté de ce Gascon qui n'avait pas l'habitude de couvrir d'éloges des guerriers huguenots?

\section{La biographie de la Noue}

Etant conscient des limites d'une étude consacrée à un seul homme, nous proposons un réexamen de la carrière et du caractère de François de La Noue, un homme qui, selon le père Lelong, "a joué un si grand rôle dans les premiers troubles de la Religion que sa Vie en est comme l'Histoire."'s

Son nom apparait dans presque tous les récits des historiens, des chroniqueurs et des mémorialistes du 16 e et du début du 17 e siècle. Si l'on repérait et rassemblait toutes les sources qui contiennent des référénces à la Noue, il serait possible de le suivre de jour en jour - et parfois même 
d'heure en heure - pendant quarante ans. Mais il est évident qu'une telle étude dépasserait de loin le cadre de cette revue, et l'on est en droit de se demander si cette énorme entreprise aiderait à mieux saisir l'intérêt essentiel de la vie de cet homme et de son oeuvre. Ainsi plutôt que d'analyser en détail chaque escarmouche, chaque bataille, chaque entretien avec un adversaire, etc., nous porterons un jugement global sur la totalité de sa vie.

Ecrivant en 1892, Henri Hauser a reconnu que, "nous sommes loin ... d'avoir pu explorer tous les dépôts qui peuvent ou même qui doivent contenir des pièces relatives à La Noue; et si cette vie présente peut-être quelques énigmes insolubles, il est aussi des lacunes que d'autres, plus heureux que nous, pourront sans doute combler." ${ }^{1}$ Hauser a suggéré, par exemple, qu'il était "peu croyable que les archives des petites villes flamandes ne conservent pas encore des lettres de La Noue." " Etant ainsi conscient des lacunes qui restaient à combler, il a pourtant conclu qu'“en présence des documents que nous avons consultés, nous ne pensons pas que des trouvailles nouvelles puissent sensiblement modifier notre récit ou altérer nos conclusions." lancés à la postérité: parviendra-t-on à mettre en doute soit son récit de la vie de La Noue soit ses conclusions sur le comportement de cet homme et sur la valeur de sa vie?

De telles questions se sont posées lors de la conception de la présente étude: les années qui se sont écoulées ont-elles confirmé le bien-fondé de la position de Hauser? Pour rendre justice à La Noue, fallait-il se lancer à la recherche d'éventuelles sources inédites cachées dans des bibliothèques isolées? Après avoir examiné et les sources du seizième siècle et les ouv rages qui ont été publiés depuis la parution de la thèse de Hauser, il nous a semblé qu'il n'y avait pas lieu de "sensiblement modifier" son récit. Si Hauser n'a pas fourni de réponses à toutes les énigmes posées par la vie de ce capitaine, il a eu le mérite d'avoir posé les bonnes questions et d'avoir établi les points de repère principaux. Quant à son analyse du personnage, on peut légitimement poser d'autres questions et proposer d'autres interprétations.

Il s'agira donc de rouvrir le dossier en utilisant le fil qu'a tissé Hauser et en tenant compte des contributions de l'historiographie du vingtième siècle. Nous nous sommes efforcé de ne pas commettre "l'erreur d'enfermer les événements dans un cercle trop mesquin, de tout ramener à la portée d'un individu, de grossir par artifice les 'questions de personnes' et de mettre les catégories générales dans la dépendance de simples incidents." 9 


\section{Le portrait de Hauser: "confiante et indulgente bonhomie"}

Chaque époque est marquée par ses préoccupations idéologiques, voire par de véritables idées fixes. Celle de Hauser avait les siennes, nous avons certainement les nôtres. Le portrait de La Noue que l'on trouve chez Hauser reflète deux obsessions du dix-neuvième siècle: le nationalisme et le laïcisme. Hauser, d'origine israélite, aurait voulu que La Noue fût un bon citoyen patriote d'une république laïque, et il semblait éprouver un besoin intense de montrer que les protestants (et, avec eux, d'autres minorités) étaient des Français à part entière. En essayant de montrer que le plus grand héros de la Réforme naissante était "un bon Français," ne cherchait-il pas aussi à prouver que tous les protestants (et peut-être aussi les Juifs) étaient à leur tour "de bons Français"?

Non que ses concitoyens du dernier quart du dix-neuvième siècle aient eu besoin de recevoir des leçons de patriotisme; au contraire, la communauté protestante cherchait à se protéger du chauvinisme exclusiviste qui s'est manifesté lors de l'Affaire Dreyfus. Comme les Juifs, les témoins protestants de la fin du siècle font état d'une "campagne d'accusations, de calomnies de toutes sortes, qui se poursuit depuis quelque temps contre nous et que cherchent à perpétuer certains journaux, certains livres. ... On fait beaucoup de bruit aujourd'hui autour de cette grande formule: $L a$ France aux Français, et l'on s'en sert contre nous." ${ }^{10}$ Il s' agit évidemment des répercussions de la montée de l'Allemagne et de l'humiliation de 1870: "Les débuts de la guerre franco-prussienne de 1870 donnèrent lieu à une recherche de coupables dont les protestants français eurent à souffrir. Une campagne de presse les accusa de souhaiter la victoire de l'ennemi, voire parfois de l'aider activement."'11

Hauser et les protestants cherchaient à repousser deux accusations. Il y avait, d'une part, celle qui dépeignait le protestantisme, enfant bâtard du "renégat saxon" Luther, comme un phénomène essentiellement étranger: "Du fait que Calvin s'était réfugié à Genève, ... que les grandes puissances, Angleterre, Allemagne, souvent ennemies de la France, étaient à majorité protestante, une suspicion s'attachait à la minorité réformée française." ${ }^{12}$ D'autre part, ils luttaient contre l'idée qu'ils devaient forcément être soupçonnés de "collaborationisme" avec les ennemis. Bien qu'ils aient fait semblant de ne pas prendre au sérieux de telles accusations - "A qui fera-t-on croire qu'il y a un péril protestant, un complot protestant, que nous voulons livrer la France à l'Angleterre ou à l'Allemagne?'13 - ils se sont crus obligés d'y répondre. L'on peut être tenté d'en sourire, mais le parallèle ne paraissait que trop évident à ceux qui rêvaient d'une France "toute catholique" et qui s'en prenaient aux "ennemis de l'âme française": ${ }^{14}$ de même que les huguenots avaient cherché l'appui d'Elisabeth et des princes allemands contre les rois Très-Chrétiens, leurs descendants étaient capables de "vendre la patrie" aux ennemis contemporains. 
Si les Rochelois avaient invité les Anglais à s'établir chez eux, si Condé avait fait venir des reîtres, si l'Etat protestant avait ébranlé le trône des Valois, que pouvait-on espérer de leurs petits-fils? Tel était le climat à l'époque où Hauser rédigeait sa thèse.

Les protestants se sentaient particulièrement exaspérés par ceux qui prétendaient qu'il y avait "une sorte d'incompatibilité entre l'esprit protestant et l'esprit français . . . que le protestantisme est contraire à notre caractère national."15 Ils insistaient donc sur les origines purement françaises de la Réforme, sur "le caractère profondément national du protestantisme français," 16 mais ce faisant ils reconnaissaient la priorité accordée aux intérêts de l'Etat-nation: "Notre Eglise est une Eglise essentiellement nationale. Et savez-nous pourquoi? parce qu'elle est protestante." 17 Le "bon" protestantisme égalait donc le nationalisme. Ils étaient fiers d'avoir contribué à la formation des institutions démocratiques grâce à "la participation des laïcs à l'administration ecclésiastique." 18 Le protestantisme qu'ils envisageaient était ainsi un protestantisme respectueux de la règle du jeu de la Troisième République: un régime laïc, démocratique, bourgeois, tolérant, exigeant toutefois la subordination de l'Eglise à l'Etat.

Hauser aurait voulu que La Noue fût conforme à cet idéal laïc et nationaliste. N'a-t-il pas fini par écrire "une étude historique où le passé pouvait soutenir l'opinion libérale dans le présent'?'19 Mais force lui était de constater que son héros s'est écarté de cet idéal à de nombreuses reprises: il a participé activement à des soulèvements armés contre l'autorité légitime de son pays; il avait des contacts avec des agents de puissances étrangères; il a fait introduire sur le territoire français des soldats étrangers; il a cherché à embrouiller des sujets français dans les affaires des PaysBas, sachant bien que cette intervention aurait pu mener à une guerre avec l'Espagne; il a collaboré avec la machine administrative de l'Etat protestant, etc. Comment la communauté protestante aurait-elle osé proposer comme héros un homme qui, au dix-neuvième siècle, aurait pu être considéré comme un traître?: "L'on ne voulait à aucun prix, dans certains milieux protestants français - surtout à Paris, centre du nationalisme, et chez les Réformés - prêter le moindrement le flanc aux accusations selon lesquelles le protestantisme était en France un élément étranger."20 (Evidemment, il est inutile d'insister sur le fait que le comportement des rois de France et des extrémistes catholiques mérite l'étiquette de "traitre," mais il s'agit ici de François de La Noue.)

Hauser se voyait donc obligé d'expliquer certains aspects "gênants" du comportement de son héros. Il s'efforce de démontrer que la politique de La Noue était dominée par un sentiment national, d'où une litanie d'affirmations de son patriotisme. Comparé à certains huguenots, La Noue était "trop bon Français et trop peu fanatique à leur gré" (xiv), et "trop bon Français pour servir d'instrument docile"' (xvii) aux desseins des Anglais. 
Il était "toujours prêt à aider la grande reine protestante, s'il pouvait le faire sans manquer à ses devoirs de Français" (p. 129; cf. pp. 282, 234, 76, 90). Comparé à Du Guesclin, La Noue "se décida, non comme un chef de bande du XIVe siècle, mais comme un bon Français du XVIe" - ne faut-il pas lire, "du XIXe siècle" (p. 268)? Hauser s'efforce de concilier religion et nationalisme: "En même temps que chrétien, il est resté Français" (p. 260 ) et "faisait passer l'intérêt national avant celui de sa secte" (p. 76), sauf après 1573 , lorsque "le politique parut l'emporter sur le chrétien, le sectaire sur le Français" (xviii). Hauser essaie d'attribuer à La Noue une espèce de "nationalisme" qui, en réalité, n'était concevable qu'après la Révolution de 1789: à La Rochelle, La Noue refuse de commettre "un crime de lèse-nation... Il possède, à un très haut degré pour un homme de son temps, et presque en dehors du sentiment loyaliste, un véritable sentiment national. ... La Noue y répond comme un moderne, qui a le sentiment très vif de la nationalité française" (pp. 288, 164, 223). Hauser insiste sur son sentiment de "fraternité nationale" (p. 245), de "solidarité nationale" (p. 286), de "devoir national" (p. 267). La Noue aurait professé les doctrines d" "une école nationale" (p. 288). Bref, à en croire Hauser, "il croit même, et c'est chose nouvelle, qu'en dehors et au-dessus de ces devoirs envers son roi, il en a, et de plus sacrés encore, envers cet être moral qui s'appelle la patrie" (p. 288). S'il est indéniable que La Noue faisait preuve d'un sentiment "nationaliste" qui mérite, à certains égards, l'étiquette de "moderne," ce genre d'explication va amener Hauser à une impasse.

S'il est embarrassé par la question de la "trahison," on sent qu'il est également gêné par la foi de La Noue. De même qu'il cherchait un patriote modèle, il aurait voulu trouver chez La Noue un protestantisme de bon ton qui ne risquât pas de choquer la sensibilité bourgeoise, un protestantisme mesuré, "raisonnable", "philosophique" qui incarnât les plus nobles aspirations de la Révolution. Et lorsque La Noue ne s'y conforme pas il faut, encore une fois, trouver une explication pour le protéger contre les accusations de fanatisme.

Si le guerrier intrépide ne s'est pas adonné au culte des Anciens, "il s'est moins bien défendu contre une autre cause d'erreur, il a cru aveuglément à la lettre de la Bible. On le voudrait un peu moins servilement attaché au texte de saint Paul, on lui souhaiterait un peu plus de cette noble indépendance d'esprit, de cette largeur de pensée qu'on rencontre chez les grands païens de l'époque, chez ces épicuriens qu'il a condamnés au nom de la foi, les Rabelais et les Montaigne" (pp. 280-81). (Notons en passant que la critique bourgeoise a essayé ainsi de récupérer Rabelais et Montaigne.) Mais si on élimine cet attachement à la lettre de la Bible, qui découle évidemment du principe de Sola Scriptura, et la doctrine fondamentale de saint Paul, la justification par la foi et par la grâce, on peut se demander ce 
qui reste non seulement de la foi de La Noue mais aussi de la Réforme. On sent que Hauser est également gêné par la foi et par le comportement des premiers huguenots: "Ce rude soldat avait en lui je ne sais quoi de bonhomme et de bon enfant; cela lui donne un visage à part, et plein d'attrait, au milieu de l'austère et un peu ennuyeuse compagnie des calvinistes" (p. 197). Il trouve que son style, "si vif et si leste à l'ordinaire, devient parfois grave et terne au point de faire déjà pressentir ce qu'on appellera plus tard le 'style réfugié'"' (p. 281). Les pasteurs sont présentés comme des fanatiques aveugles (Chapitre II). Mais il faut demander si Hauser cherche des gens qu'il aurait aimé rencontrer dans un salon parisien ou des gens prêts à tout sacrifier pour pouvoir vivre selon la pureté de l'Evangile. Estce qu'il ne finit pas par renforcer le stéréotype du protestant froid, renfrogné, sévère, etc.? L'on comprend que, vivant dans un milieu intellectuel dominé par les idées d'un Renan ou d'un Comte, Hauser ait pu se sentir gêné par cette foi trop primitive, trop naïve, trop "crue," mais cette attitude influe sur son analyse du caractère et du comportement de La Noue.

De même qu'il a insisté sur la "modernité" du seizième siècle, Hauser aurait voulu voir en La Noue un homme moderne: "Il possède, à un très haut degré pour un homme de son temps .... un véritable sentiment national. . . . La Noue y répond comme un moderne. . . . Il se décida, non comme un chef de bande du XIVe siècle, mais comme un bon Français du XVIe.... On se prend, malgré soi, à songer à ces philosophes du XVIIIe siècle qui devaient travailler au triomphe de la tolérance et de l'humanité" (pp. 164, 223, 268, 280). Mais le fait est que La Noue n'aurait pas pu se conformer à un tel idéal philosophique, laïc et nationaliste, même s'il avait pu le concevoir. Et lorsque Hauser le constate, il en est déçu. Il est indéniable que La Noue aimait profondément une entité appelée "France", que ses Discours constituent un programme pour la restaurer, et qu'il termine sa Déclaration en affirmant "Ie suis un bon François" (p. 20). Il est "moderne" à certains égards, témoin ses tendances absolutistes, centralisatrices et "tolérantistes."

Mais Hauser ne tient pas assez compte des différences sémantiques survenues au cours de trois siècles d'histoire, attribuables notamment à une Révolution qui a bouleversé les structures de l'Ancien Régime. Les mots "patrie," "nation" et "France" n'évoquaient plus pour un citoyen de la République au dix-neuvième siècle ce qu'ils évoquaient pour un sujet du Royaume au seizième siècle. La Noue utilise aussi l'expression "bon citoyen" (Observations, p. 786), mais qui oserait prétendre qu'elle avait en 1585 les mêmes résonances qu'elle aura dans la bouche d'un Robespierre, d'un Danton, d'un Saint Just? C'est toute la différence entre un "ancien" et un "nouveau" régime. Il faut surtout faire attention à des mots comme "nation" et "national" qui, après 1789, avaient pris des connotations que La Noue n'aurait même pas pu concevoir. Hauser voudrait 
que le "patriotisme" de La Noue soit le "nationalisme" post-révolutionnaire et post-napoléonien, que son protestantisme soit un protestantisme post-renanien. Mais ne finit-il pas par tomber dans le piège des antiprotestants dont l' "interprétation de l'actualité fut souvent une projection de leur vision du passé sur le présent, comme si les rapports entre religion et politique ou la conception du patriotisme, par exemple, avaient été les mêmes au XVIe siècle et au tournant du XIXe et du XXe siècles"? ${ }^{21}$ Il n'accepte pas qu'un homme du seizième siècle ait pu légitimement être tiraillé entre plusieurs devoirs contradictoires, ceux de la religion, de la féodalité, du "patriotisme" régional ou national, de l'amitié, etc. Un homme du dix-neuvième siècle ne pouvait plus l'être parce que l'évolution historique avait fait de la loyauté à l'Etat-nation la valeur suprême, et tout devait être subordonné aux intérêts de la nation (même si on prétendait ne voir aucun conflict entre les intérêts de la religion et ceux de l'Etat). Dans l'Europe d'états-nations du siècle dernier, le comportement des huguenots et de La Noue aurait pu être considéré comme un comportement de "traîtres." Mais c'est précisément parce qu'ils ne vivaient pas au dix-neuvième siècle qu'on ne peut pas les juger d'après les critères de ce siècle; il ne faut pas non plus essayer de justifier le comportement de La Noue en utilisant des critères d'un siècle qui n'était pas le sien. Cet homme qui a vécu au seizième siècle se considérait comme un "bon citoyen," mais il a commis des actes qui auraient scandalisé un "bon citoyen" du dix-neuvième siècle. Si ce genre de comportement n'était guère apprécié par les monarques du seizième siècle, La Noue n'a pas été traité avec la même rigueur que l'auraient été des ennemis de l'Etat moderne: ce rebelle a été reçu à la cour et nommé ambassadeurà La Rochelle! (Et rappelons encore une fois que le comportement des rois et des extrémistes catholiques n'était guère un modèle de probité, de bonne foi, de patriotisme.)

L'on voit le dilemme auquel a dû faire face Hauser, embarrassé par ce qu'il appelle "de véritables défaillances morales" (pp. 62, 286) de cet homme qui semble bel et bien avoir mis les intérêts de sa foi avant ceux de sa patrie, mais qui semble avoir, à d'autres moments, mal servi ses coreligionnaires et ses "bienfaiteurs," prêtant ainsi le flanc aux accusations d'un Denis d'Aussy. ${ }^{22}$ La seule explication que propose Hauser, c'est la naïveté, aussi invraisemblable que cela puisse paraitre: "Il eut ses faiblesses. Son esprit, ferme et sage en beaucoup de choses, n'était pourtant pas sans travers; le moindre était la naïveté" (p. 280). Ces termes reviennent à travers toute l'étude de Hauser: "Dans sa naïve confiance, il ne voulait croire au péril. ... sa sensibilité un peu naïve. ... on le savait naïf, scrupuleux à l'excès. . . . Il n'échappe pas à une certaine naïveté," etc. (pp. 85, 35, 225, 177). Il va encore plus loin: "Ce rude soldat avait en lui je ne sais quoi de bonhomme et de bon enfant" (p. 197) et "une candeur qui ... est bien près de toucher au ridicule" (p. 285). A en croire Hauser, La 
Noue aurait même oublié un des fondements de la doctrine calviniste (pour ne pas dire, chrétienne): "Sa confiante et indulgente bonhomie ne pouvait croire longtemps à la méchanceté, surtout à la méchanceté persévérante. ... Il est trop désintéressé. . . . Il ne croit vraiment pas assez à la méchanceté des hommes. . . . Il ne sait pas haïr" (pp. 35, 177). Hauser affirme que "La Noue, c'est son honneur et son ridicule, ne croit pas à la méchanceté humaine. Au fond, cette impossibilité de croire au mal, cette confiance exagérée dans les retours de bonté dont les pires sont parfois capables, c'est la grande, l'irrémédiable infirmité de La Noue, le seul défaut de cette intelligence si nette et si pratique, de ce ferme caractère" (pp. 283-84). C'est à cause de ces traits que La Noue ne pouvait pas se débarrasser de "la manie de la conciliation universelle" (p. 284), de "cette chimère d'universelle réconciliation"' (p. 285).

\section{Le témoignage des contemporains: d'un "iugement solide \& posé"} aux "surprinses \& meschancetez"

L'on voit que Hauser se permet d'utiliser des termes comme "ridicule", "exagéré", "infirmité", "manie", etc. pour justifier le comportement de La Noue lorsque celui-ci ne se conforme pas à l'idéal républicain et patriotique. Mais Hauser n'est pas hostile à son héros; au contraire, il admire La Noue et maintient qu'il mérite le surnom de "Bayard Huguenot." Il tente le "coup d'escrime désespéré" parce qu'il est sûr que les énormes qualités du Bras de Fer l'emporteront facilement sur ses défauts. Mais, confronté à ces "défaillances", il a recours à une explication qui, à notre avis, déforme les véritables traits de La Noue. Il n'était pas nécessaire de forcer le lecteur à prononcer La Noue coupable soit de "trahison" soit de "naïveté": il n'était pas naïf lorsqu'il négociait avec les rois, de même qu'il n'était pas traître lorsqu'il s'opposait à la politique de ceux-ci. Sans vouloir paraître désinvolte, l'on peut se demander si Hauser ne finit pas par évoquer plutôt l'image d'un pépère quelque peu gâteux en train de somnoler devant la cheminée-et qu'on réveille de temps en temps pour lui demander si on peut se fier à la parole du roi de France, s'il faut livrer La Rochelle aux Anglais ou pousser le royaume à la guerre en envahissant les Pays-Bas, si Henri de Navarre devrait se convertir au catholicisme, etc.! Le contraste entre le portrait qu'en fait Hauser et le rôle que La Noue ajoué en réalité semble démesuré. N'a-t-il pas décrit un Candide huguenot? Et pourtant, ce "bonhomme enfantin" a survécu à tous ses maîtres à travers quarante années de luttes sanglantes, acquérant une réputation qui "est sortie bien loin hors de la France, \& s'est estenduë iusques en Espagne, Italie, Allemagne \& Angleterre"'?23

Où est l'homme qui, selon ses ennemis acharnés, avait "servy de vraye phare \& guide en l'armée des heretiques, en laquelle il a tant apprins \& pratiqué de surprinses \& meschancetez, qu'il se peut aysément vanter estre 
le plus redouté de ceux qui tiennent pour le iourd'huy leur party"'?24 Auraient-ils craint à ce point un "bonhomme crédule"? Les Ligueurs qui le détestaient n'hésitaient pas pourtant à lui attribuer "une des meilleures têtes qu'il y ait" en l'armée de Henri IV. ${ }^{25}$ Son ennemi Brisson insiste sur sa maîtrise de la ruse: "La Noue pratiquoit soubz main \& s'asseuroit de ceux desquelz il se vouloit servir pour executer l'entreprise." ${ }^{26}$ Brisson en fait le portrait suivant: "Il est douë d'un esprit assez vif, d'une grace douce, qui à sa contenance monstre qu'il pense plus qu'il ne dit. ... Toutesfois ceux qui le frequentent l'ont en reputation d'estre homme d'entreprise \& d'execution, de sçavoir conduire \& mener les hommes à la guerre, d'estre brave \& adroit gentil-homme, versé és affaires d'estat. Ceux encore qui le suivent, louent en luy un iugement solide \& posé." ${ }^{27}$ L'on recherche en vain le grand benêt décrit par Hauser.

Aurait-on confié la lieutenance de la Guyenne à Candide? Aurait-on envoyé un tel député à la cour "pour recevoir les plaintes \& remonstrances de leurs confederez; afin de les faire conoistre au Roy qui leur prometoit y pourvoir selon le besoing"?28 Bentivoglio l'appelle un "personnage de grande valeur \& des plus estimez aux affaires de guerre qu'il y eust alors en France parmy la faction des Huguenots." ${ }^{29}$ Son ami La Popelinière le considérait comme "un des plus accomplis Gentils-hommes de toute la France" et "l'un des plus avisez \& resolus guerriers de France. ... II n'y avoit en France Gentilhomme de la Religion plus signalé que lui pour le maniment des armes \& affaires de consequence." ${ }^{30}$ Est-ce que les Flamands auraient confié le commandement de leur armée, et donc leur propre destin, à un naïf? J.B. de Blaes écrit que les Etats l'ont invité parce qu' "on l'estimoit le plus habile \& plus experimenté Capitaine en l'art militaire, qui fut en son temps. ${ }^{\prime}{ }^{31}$ Les ambassadeurs anglais rapportent que les ennemis refusaient parfois de se battre, fuyaient en terreur et abandonnaient des places à l'annonce de l'approche de La Noue. ${ }^{32}$ L'on peut se demander comment la capture d'un bonhomme enfantin aurait pu inspirer le désespoir chez les uns, le déchaînment de joie chez les autres, y compris le roi d'Espagne. Après la prise de Bruges, le prince de Parme s'est vu obliger de reconnaître que "La Noue . . . a enfin si bien joué son personnaige, qu'il l'a surprins par intelligence de quelques sectaires." ${ }^{33}$ (Une tentative de s'emparer de Bruxelles et du prince de Parme a échoué.) Rappelons que sa première action d'éclat avait été la prise d'Orléans "moins par la force... que par la ruse \& l'artifice; ce qu'il executa avec autant d'habileté que de bonheur." ${ }^{34}$ En 1574 il est entré à La Rochelle grâce à un coup d'état, et vers la fin de sa vie il utilisait les mêmes méthodes: "Toutes les trouppes de ces hérétiques, Givry et la Nouë, furent pour surprendre la ville de Meaux, avec l'intelligence de certains politiques de dedans, lesquels par force ouvrirent une des portes de la ville." ${ }^{35} \mathrm{De}$ Thou fournit le témoignage suivant: les ennemis ayant fait circuler de faux bruits afin d'attirer les 


\section{8 / Renaissance and Reformation}

huguenots dans une embuscade, "La Noue, qui étoit l'homme du monde le moins crédule, n'ajoûta ps beaucoup de foi à ce bruit. Les bonnes nouvelles, dit-il, qui nous viennent par la voye des ennemis, doivent toûjours nous être suspectes, \& il est bon d'être en garde contre les pièges qu'ils pourroient nous tendre." ${ }^{36}$ C'est sa prudence qui l'amenait souvent à prendre des risques "imprudents": il "retourna à la porte Saint-Michel, \& s'étant approché seul pour examiner avec plus d'attention l'endroit qu'il vouloit attaquer, il reçut au bras gauche un coup qui lui cassa l'os'"37 (cf. p. 706). Il a été tué par une balle tirée dans des circonstances pareilles. Henri de Navarre s'est moqué de lui à cause de sa prudence "excessive," mais L'Estoile raconte que La Noue, "un des plus vieux \& experimentés Capitaines de la France ... lui prédisoit ce qui en advint." 38 Même les réformés extrémistes retenaient leur jugement: "Quant à moy en telle division \& partialité d'opinions ... ie suspendi, comme ie tiens encore suspendu, mon jugement de son affaire: ne voulant rien temerairement prononcer d'un gentilhomme si bien qualifié que cestuy-là."39

Les affirmations de Hauser et des contemporains de La Noue divergent à tel point que l'on peut être tenté de demander s'il s'agit du même personnage! Seuls les réformés les plus extrémistes s'en prenaient à sa crédulité, et bon nombre d'entre eux le regrettaient après son départ de La Rochelle. A notre avis, ce sont les contemporains qui avaient raison, et nous essaierons de montrer que la naiveté est un mobile insuffisant pour expliquer le caractère et le comportement de La Noue. Bien au contraire, il y a d'excellentes raisons de conclure que sa politique n'était ni celle d'un naif ni celle d'un traître, mais plutôt celle d'un homme d'état réaliste et accompli à qui on pourrait approprier l'éloge qu'il a lui-même fait de Coligny: “'C'estoit un personnage digne de restituer un Estat affoibli \& corrompu” (p. 780). Selon nous, on diminue la grandeur et l'humanité d'un tel homme en faisant de lui un type de naif simplet. S'il s'est trompé à certains moments, ce n'est pas par naiveté ou par faute de lucidité; c'est plutôt parce qu'aucun homme dans une situation pareille n'aurait pu rassembler suffisemment de renseignements pour y voir clair. Il fallait donc peser les risques et en accepter les conséquences, et La Noue était le premier à reconnaître que "les plus fins, \& qui ouvrent bien les yeux, ne laissent quelquefois d'y estre attrappez" (p. 707).

Contrairement à ce qu'en dit Hauser, La Noue comprenait bien le monde de la guerre et de la Realpolitik; il connaissait bien les mobiles du caractère humain, notamment ceux des "Grands"; il était conscient du caractère instable et "ondoyant" des affaires humaines; et il reconnaissait la possibilité de l'échec. Mais à notre avis le secret de sa grandeur gît dans le fait qu'il n'en désespérait pas, s'étant efforcé d'accepter avec équanimité les vicissitudes de l'existence. (La sienne en a certainement eu!) Face à des obstacles qui paraissaient insurmontables, il croyait à la dignité de la lutte, 
à la possibilité de surmonter "ce qui espouvante tant de gens, \& principalement les delicats" (p. 779). Tenant compte de la "branloire perenne," il croyait à la nécessité de confronter des choix pénibles, de juger, de choisir et, une fois le choix fait, de s'y lancer corps et âme, sachant bien qu'il pourrait rencontrer l'échec, mais n'oubliant jamais qu'il pourrait se relever le lendemain pour reprendre la lutte. C'est cette "résilience" exceptionnelle, cette réaffirmation continuelle de l'esprit humain qui caractérise son attitude et explique son comportement - et qui risque de dérouter l'historien! C'est ce que Hauser prend pour de la naïveté. Mais faut-il toujours assimiler espoir, optimisme, équanimité et confiance à naïveté, crédulité et niaiserie? Le fait que La Noue s'efforçait de ne pas être méchant ne veut pas dire, comme Hauser l'affirme, qu'il était incapable de soupçonner les autres d'être méchants. Le fait que La Noue n'était pas un traître ne le rendait pas incapable de se méfier des intentions d'un Charles IX ou d'une Catherine de Médicis. L'on peut croire à la méchanceté sans être soimême méchant; l'on peut reconnaîitre l'existence du mal sans pour autant s'y abandonner. La Noue a passé toute sa vie dans ce que Montaigne appelle une "vraie eschole de trahison, d'inhumanité et de brigandage" sans pour autant devenir traître, inhumain et brigand. Il y a survécu sans perdre sa "constante bonté, douceur de meurs et facilité conscientieuse." Ces qualités ne l'ont pas pourtant empêché d'être un "grand homme de guerre et très-experimenté." 40 C'était un homme exceptionnel parce qu'il réfléchissait à ce qu'il faisait et fondait son comportement là-dessus - mais de tels hommes existent. Ne réduisons pas sa grandeur en expliquant son comportement par des "manies" ou des "chimères."

\section{La vision idéologique de La Noue: "naiveté" ou "modernité"?}

Nous espérons que cette analyse des Discours de La Noue contribuera à mettre en relief un aspect peu connu de son caractère. La Noue était dans un sens un "loyal serviteur," un "Bayard huguenot," mais il servait loyalement des principes auxquels il avait bien réfléchi, sans se laisser aveugler, sans abandonner la raison. Il réfléchissait avant d'agir en s'efforçant de conformer sa vie à des modèles idéologiques cohérents. La comparaison avec Bayard risque de le réduire à un type quelque peu béat et dépourvu de subtilité. Nous espérons avoir montré que c'était un homme intelligent, sensé, prudent et même malin. Sans ces qualités, il n'aurait pas pu survivre dans l'univers guerrier, cette "eschole de trahsion, d'inhumanité et de brigandage."

Serions-nous "naif" à notre tour de voir dans cet homme un mélange unique, l'incarnation de l'idéal noble et de l'idéal calviniste? De sa formation nobiliaire, il tire le modèle idéologique d'une vie fondée sur la poursuite de la vertu, de la justice, de l'honneur, aussi bien que le sens de mission: la défense des opprimés et du royaume de France. ${ }^{41}$ Il reconnaît 
pourtant des limites et s'efforce de maîtriser les penchants à la violence gratuite. Du calvinisme il tire sa confiance, surtout la conviction qu'il existe un plan divin et que l'homme a un rôle actif à jouer. S'il reconnaît la possibilité de l'échec, il se lance dans la bataille comme si la victoire était inévitable. S'il ne cherche pas activement la défaite, les douleurs ou la mort, il ne les craint plus: "Quand elles lui adviendront, il ne fera pas l'accident plus grand qu'il est, ains taschera, avec la vigueur de l'esprit, de le rendre encores plus petit. . . . Ils ne se contristent point outre mesure de quitter une vie caduque \& transitoire pour une parfaictement accomplie de tous bien eternels," etc. (pp. 571,580). La Noue s'efforce donc de vivre à la fois l'idéal évangélique et l'idéal guerrier.

Nous n'hésiterons pas à affirmer que c'était un homme exceptionnel qui a assuré la survie de la Réforme et qui en honore l'histoire. N'exagérons pas en faisant de lui un saint, car son idéologie comporte des éléments qui choqueront (espérons-le) le lecteur "éclairé" du vingtième siècle. Mais s'il est indéniable qu'il accepte le principe d'une société fondée sur le privilège, il insiste quand même sur la nécessité de mériter ces privilèges et se montre tout à fait insensible à l'égard de ceux qui trahissent leur vocation "naturelle" de cadres dirigeants. S'il consent à peine à reconnaître l'humanité du "peuple champestre" qui semble incapable des "exercices superieures de l'ame" ("Mais la charité nous doit faire juger que Dieu ne fait rien en vain," p.606), il n'hésite jamais à risquer sa vie pour le défendre contre les atrocités perpétrées par ses pairs de la noblesse, ces "harpyes militaires." S'il est également indéniable qu'il recourt à la force et à la violence pour défendre une foi fondée sur "misericorde," "charité" et "douceur" (p. 398), il fait tout pour en éviter l'emploi et s'efforce toujours d'en minimiser les dégâts, car "l'homme doit principalement tendre à paix \& tranquillité, à fin de mener une vie plus juste" (p. 210. Ajoutons en passant que les idéologies "progressistes" et "scientifiques" qui ont succédé aux doctrines "réactionnaires" de l'Ancien Régime se sont avérées beaucoup plus sanguinaires et répressives.) A notre avis, le mot "grandeur" n'est pas déplacé, une grandeur qui provient moins de ses exploits militaires que de son caractère et de sa capacité de réflexion. Il se montre toujours plus exigeant à l'égard de sa propre conduite qu'à celle de ses contemporains et se croit d'autant plus autorisé à fustiger ceux-ci qu'il s'efforce consciemment de réaliser une synthèse entre l'idéal nobiliaire et l'idéal évangélique. Il s'en est approché.

Quelle est alors la valeur du témoignage vécu et écrit que nous a laissé La Noue? Comme il serait téméraire et déplacé de vouloir empiéter sur le domaine de la foi personnelle des Réformés du vingtième siècle en leur proposant une réponse à cette question, nos conclusions s'organiseront plutôt autour de deux concepts qui risquent de prêter à des malentendus: la "modernité" et la "naïveté." Non que ces concepts entrent forcément en 
contradiction l'un avec l'autre; mais comme Henri Hauser s'en sert, non seulement dans son étude sur La Noue, mais aussi dans La modernité du seizième siècle, nous les reprenons ici pour tenter de répondre aux questions suivantes: les analyses de La Noue ont-elles anticipé sur l'avenir? Si oui, nous pourrons employer l'étiquette "moderne." D'un autre côté, La Noue ne conçoit-il pas les rapports entre les êtres humains et la nature de l'Etat sous un angle "naif," c'est-à-dire sans tenir compte de ce que Machiavel appelle la realtà effetuale delle cose? Si nous hésitons à utiliser le terme "précurseur" de peur de tomber dans les pièges de l'anachronisme ou d'une vision linéaire de l'histoire, il faut tout de même reconnaître que La Noue devance à certains égards la majorité de ses contemporains. Sa conception de l'Etat et sa vision de l'avenir de la noblesse illustreront cette thèse.

Prenons d'abord sa conception de l'Etat en en analysant trois aspects complémentaires: le nationalisme, le monarchisme et la tolérance. Comme il vit à une époque de crise, La Noue propose souvent des solutions provisoires dictées par l'actualité et destinées à rétablir l'ordre le plus vite possible; mais ces remèdes "ponctuels" découlent en réalité de principes abstraits que La Noue s'est formés, soit à partir de ses lectures soit pendant sa carrière militaire. Loin de voir des contradictions entre le théorique et l'universel, d'une part, le pratique et le vécu, de l'autre, La Noue insiste sur leur complémentarité. Son sentiment national en fournit un exemple. Une des sources de son patriotisme est le danger immédiat représenté par l'Espagne. Il est d'autant plus "nationaliste" qu'il craint la domination étrangère, et la peur de voir triompher les forces du roi très-catholique joue un rôle décisif dans ses appels à l'unité nationale. (Il n'hésite pas toutefois à exprimer son admiration pour certaines qualités espagnoles: discours XIV-XVII). Mais à ce sentiment négatif fondé sur la crainte s'ajoute un réel sentiment de fierté à l'égard de la France. Il s'agit d'une attitude "moderne" en ce sens que La Noue dépasse le sentiment tribal primitif, la crainte primordiale de l'Autre en se déclarant prêt à reconnaître comme compatriotes tous ceux qui se réclament de la France: "Aux termes où est maintenant nostre Estat, un Italien francizé est bien autant à priser qu'un François espagnolizé" (p. 107). Peu de ses coreligionnaires se montrent aussi accueillants envers les compatriotes de la Reine-mère. (L'on verra qu'il s'agit naturellement d'un Etat multi-confessionnel.) Si Hauser a tort de chercher chez La Noue une espèce de nationalisme post-révolutionnaire, il a raison d'insister sur sa conscience nationale "moderne."

Ce sentiment est inséparable chez lui d'un sentiment monarchiste que l'on pourrait qualifier de "pré-" ou de "proto-" absolutiste, car La Noue tient à ce que ses compatriotes restent "affectionnez à se maintenir unis souz l'authorité de ceste couronne"' (p.415). De nouveau, théorie et pratique se réjoignent. Un roi fort lui paraît être le remède immédiat aux 
désordres causés par les guerres de religion, et ce roi aurait pu en même temps protéger les Réformés. A ces soucis immédiats s'ajoutent les exhortations bibliques qui exigent la soumission aux "magistrats" (discours X), et La Noue est conscient des dangers que comporte tout "changement de police." Qu'il ait cherché des solutions dans la science politique grécoromaine, dans l'enseignement paulinien ou dans la réalité concrète qui l'entoure, il a trouvé la même réponse: la fidélité à un roi fort qui, seul, exerce la souveraineté à la tête d'un Etat-nation unifié. Lorsqu'il se voyait obligé de s'opposer à la politique des fils de Henri II, son but n'était ni la destruction de la monarchie ni la mort des monarques: il cherchait plutôt à les ramener à la raison, à les obliger à négocier, à accorder la liberté de conscience - d'où sa modération, sa mansuétude, ses efforts d'empêcher des atrocités qui n'auraient fait que provoquer des représailles de la part des catholiques plus nombreux. Il ne voulait pas ravager le pays qui devait un jour servir à tous, réformés et catholiques. Il se comportait donc comme un homme d'état qui voyait loin, qui savait pourquoi il se battait et qui n'oubliait jamais que la fin ne justifie pas les moyens.

La politique de tolérance préconisée par La Noue relève, elle aussi, de facteurs concrets et de principes abstraits. Il est évident qu'une telle politique aurait assuré la sécurité des Réformés, permis le retour des exilés et autorisé le prosélytisme, grossissant ainsi les rangs du parti. Il n'y a donc pas lieu de s'étonner de l'attitude de La Noue. Mais il ne s'agit pas simplement de la politique intéressée d'un sectaire, car La Noue respecte la dignité inhérente de l'individu et reconnaît la suprématie de la conscience en matière de foi (bien qu'il méprise l'Islam et qu'il maintienne que "le feu est pour les Sodomites," p. 124), pourvu que le "faux zelle" ne pousse pas le croyant à répandre le sang d'autrui. L'Etat monarchiste devrait s'en porter garant, "\& si la paix regne quelque temps on verra qu'en la Chrestienté ne se trouvera de meilleurs catholiques et évangeliques qu'en France" ( $p$. 409). La coexistence de deux ou de plusieurs confessions se présente ainsi comme un moyen de contribuer à la gloire du royaume de France, d'ajouter une pierre précieuse à la couronne du roi. Hauser a donc des raisons solides de défendre l'authenticité de la "Lettre sur la conversion du Roy."42

Bien que les acquis de l'Edit de Nantes aient été progressivement rognés par les successeurs de Henri IV, les trois principes qui viennent d'être analysés constitueront la base de l'Etat français du XVIIe et du XVIIIe siècles. Dans la perspective de La Noue, l'unité nationale paraît inconcevable sans la présence d'un roi fort qui, appuyé par une noblesse régénérée, fût capable de veiller sur les droits de tous ses sujets, quelle que soit leur confession. Un tel pays pourrait alors tenir tête à d'éventuelles menaces venues de l'étranger: "Mal-aisément nous pourroient-ils ruïner en quelque estat que nous soyons, moyennant que nous demourrons en 
l'obeissance de la couronne"' (p. 435). L'analyste moderne y verra sans doute la soumission de la religion aux intérêts de l'Etat, mais La Noue n'y voit aucun conflit, ayant toujours trouvé une parfaite correspondance entre sa foi et son patriotisme. La conception de l'Etat qui se dégage des Discours préfigure ainsi certains principes fondamentaux de l'Etat absolutiste du XVIIe et du XVIIIe siècles, et de l'Etat "libéral" fondé sur la coexistence de plusieurs confessions et de plusieurs courants d'opinion. Est-ce que La Noue a aussi bien prévu le rôle que jouerait dans cet Etat le Second Ordre?

La Noue comprend bien que la France est en danger parce que ses défenseurs sont incompétents, irresponsables et "indignes de porter ces deux beaux titres de chrestien \& de gentil-homme" (p. 697). En tant que noble, il cherche à agir dans un domaine qui relève de sa compétence, ayant conclu que le salut du royaume passerait par le salut de la noblesse. Si de nombreux contemporains éprouvent un besoin urgent de réformes profondes au sein du Second Ordre, c'est La Noue qui, dans ses Discours, fournit des analyses parmi les plus perspicaces, proposant des solutions concrètes et réalisables: "Ce ne sont point icy des Idees de Platon (c'est à dire des choses imaginees)" (p. 313).

Comme ses idées sur la nature de l'Etat, ses idées sur l'éducation relèvent et du théorique et du pratique. Le spectacle effroyable des guerres de religion l'a convaincu des résultats catastrophiques de l'ignorance: ceux qui troublent "l'ordre public" et qui "s'emancipent à telles choses, le font par defaut de bonne nourriture" (p. 157). L'éducation dans des académies "écuméniques" aurait donc servi à mettre fin aux désordres et à arrêter la destruction de la noblesse. ${ }^{43}$

Mais en même temps "l'institution à pieté \& vertu" (p. 142) lui paraît nécessaire si le noble doit réaliser pleinement toutes ses virtualités. Cette insistance sur l'épanouissement moral, spirituel et intellectuel de l'homme laïc-impliquant même la contemplation et la méditation - est à mettre en rapport avec le principe réformé du sacerdoce universel: le père, "prêtre" chez lui, doit être capable de lire l'Ecriture, de l'enseigner à ses enfants, d'en tirer des leçons et d'agir en conséquence. L'union avec la Divinité est non pas un droit, mais un devoir fondamental de tout être humain, notamment du noble, né avec "des inclinations plus vives \& ployables que les autres. ... à quoy leur condition noble les doit aussi exhorter" (pp. 59596). L'on voit donc comment religion et conscience de classe se renforcent. La Noue préconise ainsi la formation de cadres laïcs dignes de diriger le royaume.

L'on comprend facilement la nécessité de bien former la faculté du jugement. La vie de La Noue confirme que le noble est confronté à des choix épineux: la nature de l'engagement du sujet dans la vie de la république; la définition d'une guerre "juste"; les limites acceptables de la force et de la 


\section{4 / Renaissance and Reformation}

violence; le devoir de refuser d'obéir à des ordres injustes; les alliances politiques "impures," etc. La religion de La Noue n'exige pas la démission intellectuelle ou la croyance aveugle des pasteurs de La Rochelle. Ses Discours et ses Observations veulent aider le noble à aiguiser ses facultés de discernement: "Cela est apprendre à estre capitaine. . . . à fin que ceux qui veulent s'instruire aux armes en tirent ce fruict. ... Quand quelque fait est descrit à la verité, $\&$ avec ses circonstances, encore qu'il ne soit parvenu qu'à my chemin, si peut-on tousjours en tirer du fruict" (pp. 661, 724, 736), etc. Son oeuvre et sa vie constituent un modèle vivant de cette pédagogie nouvelle visant à assurer la survie et l'épanouissement de la noblesse d'épée: 'La jeunesse ayant esté ainsi instituee, il ne faudroit point craindre de l'envoyer apres par tout où l'on voudroit, par ce qu'elle seroit à l'espreuve, \& au lieu de se gaster, elle iroit choisissant ce qui est de meilleur ailleurs, pour y profiter" (p. 158). Que peut-on dire de ses projets, de sa vision de l'avenir de la noblesse?

Si La Noue a bien compris la nécessité de réformes profondes au sein du Second Ordre, il ne pouvait pas concevoir la domestication de l'aristocratie accomplie au cours du XVIIe siècle. Tandis que son désir de fournir à la noblesse d'épée un nouveau type de formation intellectuelle représente une percée vers la modernité, son espoir de voir se renforcer l'harmonie entre une noblesse plus "reconnaissante" et la monarchie paraît naïf. Mais qui aurait pu prévoir que les processus de "raffinement" impliquerait aussi l'apprivoisement intellectuel, moral et même financier du Second Ordre?

$\mathrm{Sa}$ conception paternaliste de la monarchie ("Le prince ... est pere commun de ses sujets," p. 152) l'empêche de concevoir qu'un père puisse chercher à restreindre l'épanouissement de ses "fils." Il faut dire en même temps que La Noue a sous-estimé la rôle qu'allait jouer le Tiers Etat dans cette nouvelle société. Son programme préconise la reprise en main des fonctions clés de l'Etat par ceux dont les ancêtres, "estans parvenus à grandeur \& honneur, par les voyes de vertu tant intellectuelle que morale, ... leur ont laissé des petites semences d'icelles . . . aptes à les renouveller en eux"' (p. 595), etc. Mais l'évolution historique n'a pas exaucé ses voeux: il se serait certainement réjoui de voir l'aristocratie raffinée du XVIIe siècle; il se serait sans doute étonné de constater jusqu'à quel point elle s'était soumise à la monarchie et s'était adonnée au "libertinisme" dénoncé dans le discours XXIV.

Terminons donc en soulignant encore la richesse et la diversité de l'oeuvre de La Noue. On a vu que l'histoire littéraire a relégué notre auteur au rang des "chroniqueurs" ou des "mémorialistes," destin qui n'est pas dépourvu d'honneur mais qui ne rend pas justice au Bras de Fer. S'il est indéniable que le contenu des "Observations" explique ce jugment de la critique, La Noue dépasse de loin les Montluc, les Castelnau, les Barnaud, etc. Ce sont tous des témoins qui, en tant que tels, ont joué un rôle indis- 
pensable dans la compréhension de l'histoire du seizième siècle. Mais ces témoins s'avèrent souvent bornés et intéressés, cherchant à se vanter ou à justifier leurs méfaits. Sans vouloir nier que La Noue cherche à se justifier - "Il estoit tres-necessaire alors ... de lever les mauvaises impressions qui se pouvoyent prendre par ceux qui ignoroyent les intentions des entrepreneurs.... Qui ne rembarre les calomnies ... sans doute il se verroit souvent supprimé," p. 622 - nous soutenons que ce n'est pas là l'essentiel.

Tout en étant un témoin objectif et digne de foi, La Noue est avant tout un penseur, un moraliste qui cherche à renvoyer ses lecteurs à des valeurs atemporelles: le vécu et l'immédiat doivent toujours être transcendés et déboucher sur l'abstrait, l'universel et l'éternel. Que ce soit dans ses "mémoires" ou dans ses "opuscules," l'auteur s'efforce de dépasser l'éphémère en avertissant le lecteur de l'existence d'autre chose, d'une leçon générale à en tirer. Tout fait concret débouchant ainsi sur l'universel, le lecteur se voit doublement récompensé de ses efforts. C'est cet aspect de son oeuvre qui l'élève au-dessus aussi bien des mémorialistes que des polémistes déchaînés dont l'oeuvre était destinée à la "consommation" immédiate. Ainsi les véritables confrères de La Noue se nomment-ils Machiavel, Plutarque, Guichardin, Montaigne, Charron, Pascal.

Cette constatation nous mène à poser une question paradoxale: faut-il regretter que La Noue n'ait pas eu le temps de se consacrer à la réflexion et à l'écriture? D'une part, il peut paraitre regrettable que ses campagnes militaires incessantes ne lui aient pas laissé le loisir de s'adonner aux activités intellectuelles. La suite des "Observations," par exemple, serait sans aucun doute un document des plus précieux. Ses années de captivité ont été "utiles" dans la mesure où elles lui ont offert une période de temps "libre" pendant laquelle son âme était "desliee des ceps \& liens mondains" (p. 598). C'est précisément ce mélange d'action et de réflexion chez La Noue qui fait le caractère unique du personnage et de l'oeuvre, c'est son engagement personnel quijustifie son oeuvre. Ses idées ne sont pas seulement abstraites mais sont en prise sur le réel et l'action. Il se compare lui-même à Saint Augustin, qui "a approuvé du tout ceste bien ordonnee composition" de vie active et de vie contemplative, aussi bien qu'à saint Paul, qui "avec ses hautes \& profondes speculations n'a point laissé d'estre en action perpetuelle pour l'edification de l'Eglise"' (ibid.). C'est dans ce désir maintes fois exprimé de réaliser une synthèse entre le spirituel et le matériel, le divin et l'humain que réside la "noblesse" de La Noue. Il a cru pouvoir aider la classe dirigeante du royaume de France à atteindre cet idéal à travers son exemple personnel, par son engagement actif et par ses écrits. Il a contribué de la sorte au progrès intellectuel de la noblesse française, ainsi qu'à la "défense et illustration" de l'Eglise Réformée, et ce avec la plume d'un authentique écrivain dont la prose à enrichi la langue française du XVIe siècle. 
Notes

1 Le lecteur trouvera des renseignements sur la vie de La Noue dans Brantôme, $C$ Euvres, éd. Ludovic Lalanne (Paris: J. Renouard, 1873), VII: 203-65; dans Moise Amyraut, La vie de François, seigneur de la Nouē, dit Bras-de-Fer (Leyde: Jean Elsevier, 1661); et dans Henri Hauser, François de La Noue (Paris: Hachette, 1892). Ce sont des témoignages de valeur inégale: si Brantôme laisse échapper son sentiment de jalousie à l'égard de La Noue, Amyraut ne résiste pas à la tentation hagiographique. C'est donc l'étude de Hauser qui s'avérera la plus utile.

2 François de La Noue, Discours politiques et militaires, éd. Frank E. Sutcliffe (Genève: Droz, 1967). Toutes les citations seront tirées de cette édition et apparaitront dans notre texte. Comme le professeur Sutcliffe n'a pas précisé les principes dont il s'est servi pour établir son texte, nous avons comparé l'édition de 1967 à celle de 1587 ([Bâle ou Genève]: François Forest, 1587; Bibliothèque Nationale R. 6332). Lorsqu'il ne s'agit que de variantes d'orthographe qui ne mettent pas en cause le sens du texte, nous ne nous sommes pas cru obligé de les signaler au lecteur, afin de ne pas encombrer inutilement notre étude.

3 Parmi les travaux récents consacrés à La Noue, on peut citer: William $\mathrm{H}$. Huseman, "François de La Noue, la dignité de l'homme et l'institution des enfant nobles: contribution à l'étude de l'humanisme protestant", Bibliothèque d'Humanisme et Renaissance 42 (1980), 7-25; Ian Morrison, "The Dignity of Man and the Followers of Epicurus: The View of the Huguenot François de La Noue," BHR 37 (1975), 421-29; Paul Rousset, "Un huguenot propose une croisade: le projet de François de La Noue, 1580-1585," Revue d'Histoire Ecclésiastique Suisse 72 (1978), 333-44; "L'idéologie de croisade dans les guerres de religion au XVIe siècle," Revue Suisse d'Histoire 31 (1981), 174-84; James Supple, "François de La Noue's Plan for a Campaign Against the Turks," BHR 41 (1979), 273-91; "The Role of François de La Noue in the Siege of La Rochelle and the Protestant Alliance with the Mécontents, "BHR 43 (1981), 107-22; et "François de La Noue and the Education of the French Noblesse d'épée," French Studies 36 (1982), 270-81.

Parmi les chercheurs qui citent longuement La Noue, on peut mentionner André Devyver, Le sang épuré. Les préjugés de race chez les gentilshommes français de l'Ancien Régime (Bruxelles: Editions de l'Université de Bruxelles, 1973); Arlette Jouanna, L'idée de race en France au XVIe et au début du XVIIe siècle (1498-1614), (Paris: Champion, 1976); Miriam Yardeni, La conscience nationale en France pendant les guerres de religion (Louvain: Nauwelaerts, 1971); Roger Chartier, Marie-Madeleine Compère, et Dominique Julia, L'éducation en France du XVIe au XVIIIe siècle (Paris: SEDES, 1976); et René Bady, L'homme et son "institution" de Montaigne à Bérulle, 1580-1625 (Paris: Les Belles Lettres, 1964).

4 Montaigne, Essais, éd. Pierre Villey (Paris: Félix Alcan, 1922), II: 448 ("De la présomption," II, 17).

5 Jacques Lelong, Bibliothèque historique de la France (Paris: Jean-Thomas Hérissant), II: 339. Lelong cite le père Gabriel Daniel, Histoire de France depuis l'establissement de la monarchie françoise dans les Gaules (Paris: J.-B. Delespine, 1713), III: 1531.

6 Hauser, p. 274.

7 Ibid., xvii.

8 Ibid., xvii-xviii.

9 Lucien Romier, Le royaume de Catherine de Médicis. La France à la veille des guerres de religion (Paris: Perrin, 1925), I: $x$.

10 H.-J. Messines, Protestants et Français (Paris: s. éd., [1899]), pp. 3, 8. Cet ouvrage sans valeur "scientifique" s'avère néanmoins extrêmement utile dans la mesure où il exprime l'inquiétude profonde de la communauté protestante.

11 Jean Bauberot, "La vision de la Réforme chez les publicistes antiprotestants (fin XIXe-début $\mathrm{XXe}$ )," in Historiographie de la Réforme, éd. Ph. Joutard, Actes du colloque du 22-24 septembre 1972 (Neuchâtel-Paris: Delachaux \& Niestlé, 1977), p. 216. La revue Histoire a consacré un numéro aux protestants français: janvier-mars $1981, n^{\circ} 7$.

12 Ibid., "Avant-propos" de P. Guiral, p. 5 ; cf. Messines, p. 5.

13 Messines, p. 3. 
14 F. Brunetière, Les ennemis de l'âme française (Paris: J. Hetzel, s.d.), pp. 55-74; Ch. Buet, Les mensonges de l'histoire (Lille: J. Lefort, 1885-1889), I, 53-195; II, 143-86; 209-75; Edouard Drumont, La France juive (Paris: Marpon \& Flammarion, 1886); Ch. Merki, L'Amiral de Coligny, la maison de Châtillon et la révolte protestante, 1519-1572 (Paris: Plon-Nourrit, 1909); E. Renauld, Le péril protestant (Paris: Tolra, 1899); et Georges Thiébaud, Le parti protestant (Paris: A. Savine, 1895).

15 Messines, p. 4.

16 Ibid., p. 13.

17 Ibid., p. 23.

18 Ibid., pp. 25-32.

19 Daniel Robert, "Patriotisme et image de la Réforme chez les historiens protestants français après 1870," in Joutard, p. 207. Voir aussi un article typique de N. Weiss, "La prétendue trahison de Coligny," BSHPF 49 (1900), 37-47, ou les Actes du colloque Les protestants dans les débuts de la Troisième République (1871-1855), ed. André Encrevé et Michel Richard (Paris: Société de l'Histoire du Protestantisme Français, 1979).

20 Robert, p. 215.

21 Bauberot, p. 222.

22 Denis D'Aussy, “Un Bayard calviniste: François de La Noue et ses dernières campagnes," Revue des Questions Historiques 42 (octobre 1887), 397-440. L'on peut consulter aussi ses articles dans la Revue de la Saintonge et de l'A unis, 8 (juillet 1888), 280-83 et 8 (septembre 1888), 331-33; et 13 (janvier 1893), 22-34. N. Weiss lui a répondu dans le BSHPF 36 (1887), 667-78 et 37 (1888), 335-36, 388-89. Hauser a défendu l'authenticité des Discours dans la Revue Historique 53 (septembre-octobre 1893), 301-11.

23 Pierre de Dampmartin, La Fortune de la Cour (Paris: Nicolas de Sercy, 1644), p. 173.

24 La coppie d'une lettre envoyee par un gentilhomme, de l'armee de Monseigneur le Duc de Mayenne, aux Bourgeois \& habitans de la Ville \& Faubourgs de Paris (Paris: Pour Anthoine du Brueil, 1589), pp. 4-5.

25 "Discours bref et véritable des choses plus notables arrivées au siège mémorable de la renommée ville de Paris \& défense d'icelle," in Mémoires de la Ligue (Amsterdam: Arkstée \& Merkus, 1758), IV: 282.

26 Pierre Brisson, Histoire et vray discours des guerres civilles és pays de Poictou, Aulnis, autrement dit Rochelois, Xainctonge, \& Angoumois depuis l'annee mil cinq cens soixante \& quatorze (Paris: Iacques du Puy, 1578), f. Avi verso.

27 Ibid., f. Cvii recto.

28 La Popelinière, Henri Voisin, sieur de, Histoire de France (La Rochelle: Abraham H., 1581), tome second, livre 24, f. 5 recto.

29 Bentivoglio, Guido, Cardinal. Histoire générale des guerres de Flandres, trad. Antoine Oudin (Paris: François Promé, 1699), tome I, livre vi, p. 323.

$30 \mathrm{La}$ Popelinière, tome second, livre 32, f. 118 verso.

31 Emanuel van Meteren, L'histoire des Pays-Bas, trad. Jean de La Haye (La Haye: Hillebrant Jacobs, 1618), f. 156d (14 juillet 1578).

32 Calendars, avril 1579, n०668, p. 501; 10 mai 1579, n०675, p. 507; 5 octobre $1579, n^{\circ} 59$, p. 68 ; 22 novembre 1579, n० 96, p. 98.

33 Alexandre Farnèse, prince de Parme, Correspondance. . . dans les années 1578, 1579, 1580 et 1581, éd. M. Gachard (Bruxelles: C. Muquardt, 1853), p. 112.

34 De Thou, Histoire universelle (La Haye: Scheurleer, 1740), tome quatre, livre 42, p. 18.

35 La résistance des habitans de la ville de Meaux, contre les trouppes de Givry, \& la Nouè, \& leurs associez politiques (Paris: Hubert Velu, 1589), p. 7. Palma-Cayet rapporte le conseil suivant qu'a donné La Noue à Henri IV: "Nous y perdrions temps et moyens, mais peu à peu, usant des ouvertures que je feray, vous verrez que ce grand party se dissipera en soy-mesmes, et nous donnera beau jeu sans beaucoup travailler; mais il faut de la patience et de la finesse" (Chronologie novenaire, éd. Petitot (Paris: Foucault, 1824), XXXIX: 329. C'est nous qui soulignons.) 


\section{8 / Renaissance and Reformation}

36 De Thou, tome quatre, livre 47, p. 316.

37 Ibid., p. 321.

38 Pierre de L'Estoile, Journal pour le règne de Henri IV, 1589-1600, éd. Louis-Raymond Lefèvre (Paris: Gallimard, 1948), p. 72 (août 1590).

39 Nicolas Barnaud, Le réveille-matin des François, et de leurs voisins. Composé par Eusèbe Philadelphe Cosmopolite, en forme de dialogues. (Edimbourg: Iaques Iames, 1574), II: 132.

40 Montaigne, II: 448.

41 Cf. La Noue, Déclaration, p. 3: "Le devoir d'un gentilhomme faisant profession de vertu, gist en premier lieu, à si bien preparer \& digerer ses actions, qu'il en reçoive contentement en soy mesme. Il doit apres les faire reluire \& les iustifier en sorte, que les bons soient satisfaits, \& les mauvais n'ayent suiect de les condemner."

Palma-Cayet (XL: 292) raconte que, vers la fin de sa vie, La Noue a coupé "deux petites branches de laurier," et "ayant amenuisé l'une de ces branches, il la mit à son armet au lieu de pannache." En voyant entrer le sieur de Montmartin, "il luy monstra son armet entouré de lauriers, et luy dit: "Tenez, mon cousin, voylà toute la recompense que vous et moy esperons, suivans le mestier que nous faisons."

42 Voir son article "François de La Noue et la conversion du roy," Revue Historique 36 (mars-avril 1888), 311-23.

43 Voir les études de Huseman, de Morrison, de Supple, de Jouanna, et de Bady citées dans la note 3 ci-dessus. 